\section{Nuclear Physics A}

www.elsevier.com/locate/procedia

\title{
Pre-equilibrium dynamics and heavy-ion observables ${ }^{\sqrt{2}}$
}

\author{
Ulrich Heinz and Jia Liu \\ Physics Department, The Ohio State University, Columbus, OH 43210, USA
}

\begin{abstract}
To bracket the importance of the pre-equilibrium stage on relativistic heavy-ion collision observables, we compare simulations where it is modeled by either free-streaming partons or fluid dynamics. These cases implement the assumptions of extremely weak vs. extremely strong coupling in the initial collision stage. Accounting for flow generated in the pre-equilibrium stage, we study the sensitivity of radial, elliptic and triangular flow on the switching time when the hydrodynamic description becomes valid. Using the hybrid code IEBE-VISHNU [1] we perform a multi-parameter search, constrained by particle ratios, integrated elliptic and triangular charged hadron flow, the mean transverse momenta of pions, kaons and protons, and the second moment $\left\langle p_{T}^{2}\right\rangle$ of the proton transverse momentum spectrum, to identify optimized values for the switching time $\tau_{s}$ from pre-equilibrium to hydrodynamics, the specific shear viscosity $\eta / s$, the normalization factor of the temperature-dependent specific bulk viscosity $(\zeta / s)(T)$, and the switching temperature $T_{\mathrm{sw}}$ from viscous hydrodynamics to the hadron cascade URQMD. With the optimized parameters, we predict and compare with experiment the $p_{T}$-distributions of $\pi, K, p, \Lambda, \Xi$ and $\Omega$ yields and their elliptic flow coefficients, focusing specifically on the mass-ordering of the elliptic flow for protons and Lambda hyperons which is incorrectly described by VISHNU without pre-equilibrium flow.
\end{abstract}

Keywords: collective flow, pre-equilibrium dynamics, quark-gluon plasma, viscosity, model-to-data comparison, parameter optimization, uncertainty quantification

\section{Introduction}

Relativistic viscous hydrodynamics has become the workhorse of dynamical modeling of ultra-relativistic heavy-ion collisions. However, hydrodynamics does not become valid until the medium has reached a certain degree of local momentum isotropization [2]. In an inhomogeneous system, collective flow (i.e. space-momentum correlations) begins, however, to develop already before hydrodynamics becomes valid. The hydrodynamic stage thus starts with a nonvanishing pre-equilibrium flow [3-7]. In [8] we therefore performed a systematic study of preequilibrium flow effects on heavy-ion collision observables. We here summarize the main results of this study and add a few recent results that go beyond the work reported in [8].

\footnotetext{
${ }^{\star}$ Work supported by the DOE, Office of Science, Office of Nuclear Physics under Award No. DE-SC0004286. Computing resources provided by the Ohio Supercomputer Center. Thanks to Chun Shen for fruitful discussions.
} 


\section{The model}

We assume longitudinal boost-invariance and model the approach towards local thermal equilibrium in a heavy-ion collision very simply [8] as a pre-equilibrium stage of noninteracting free-streaming massless partonic degrees of freedom, separated by a switching time $\tau_{s}$ from a strongly coupled quark-gluon plasma (QGP) stage in which frequent collisions isotropize the local momentum distribution sufficiently quickly that it can be described by viscous relativistic fluid dynamics. The variable switching time $\tau_{s}$ parametrizes the duration of the thermalization process. For massless degrees of freedom the evolution of the energy-momentum tensor during the free-streaming stage is independent of the initial transverse momentum distribution as long as it starts out locally azimuthally symmetric, and can be solved analytically [8].

At $\tau_{s}$ we Landau-match the analytically evolved pre-equilibrium energy-momentum tensor to viscous hydrodynamic form [8]. Space-momentum correlations established by the freestreaming dynamics in the pre-equilibrium stage manifest themselves as non-zero initial values for the hydrodynamic flow velocity profiles, giving rise to non-zero initial radial and anisotropic flows. Local momentum anisotropies resulting from the free-streaming evolution of a spatially inhomogeneous initial density profile generate nonzero initial values for the shear stress. Matching the traceless pre-equilibrium energy-momentum tensor of noninteracting massless degrees of freedom to a non-conformal, lattice QCD based equation of state [9] at $\tau_{s}$ generates a non-zero initial bulk viscous pressure field in the hydrodynamic fluid. All of these initial fields are then further evolved with the second-order viscous relativistic fluid dynamics code VISH2 +1 .

After hadronization of the QGP at a pseudocritical temperature $T_{\mathrm{c}} \simeq 155 \mathrm{MeV}$ (determined by the equation of state [9]), the medium constituents are color neutral hadrons whose interactions are weaker than the gluon-mediated interactions in the earlier color-deconfined QGP, causing a rapid growth of their mean free paths and a subsequent breakdown of the fluid dynamic framework. We therefore switch from VISH2+1 to a microscopic description, using the hadron cascade code URQMD, on an isothermal switching surface of temperature $T_{\text {sw }}$ which we here consider as an unknown parameter to be optimized by comparison with experimental data.

Two additional important parameters affecting the evolution of collective flow and the $p_{T}$ distributions of the finally emitted hadrons are the shear and bulk viscosities during the liquid QGP stage. For simplicity, we assume the QGP specific shear viscosity $\eta / s$ to be a temperatureindependent, adjustable constant. For the specific bulk viscosity $\zeta / s$, which is expected to develop a strong peak due to critical scattering close to the quark-hadron phase transition, we adopt the temperature-dependent parametrization given in[10] (which features a peak at $T_{\text {peak }}=$ $180 \mathrm{MeV}$ ), but consider its normalization as a freely tunable parameter. In practice, peak values of $\zeta / s$ exceeding 1 (corresponding to bulk viscosity normalization factors $>3$ ) cause the (negative) bulk viscous pressure to become very large near $T_{\text {peak }}$, leading to negative total pressures and mechanical instability of the medium against cavitation. These physical instabilities also eventually render the hydrodynamic code numerically unstable.

\section{Results}

We found in [8] that an extended pre-equilibrium stage increases the final radial flow, leading to flatter $p_{T}$-spectra, while leaving the integrated charged hadron $v_{2}$ and $v_{3}$ almost unchanged unless $\tau_{s}$ significantly exceeds $2 \mathrm{fm} / c$. Bulk viscosity inhibits radial flow build-up, so the constraint from the measured $p_{T}$ distributions causes a strong positive correlation between $\tau_{s}$ and the bulk viscosity normalization factor. The experimental charged hadron elliptic flow constraint anticorrelates bulk and shear viscosity; at low $p_{T}$, the bulk viscous correction $\delta f_{\text {bulk }}$ at $T_{\mathrm{sw}}$ acts 
as an effective positive chemical potential for massive hadrons [12], causing an anticorrelation between the bulk viscosity normalization factor and the chemical decoupling temperature $T_{\mathrm{sw}}$.
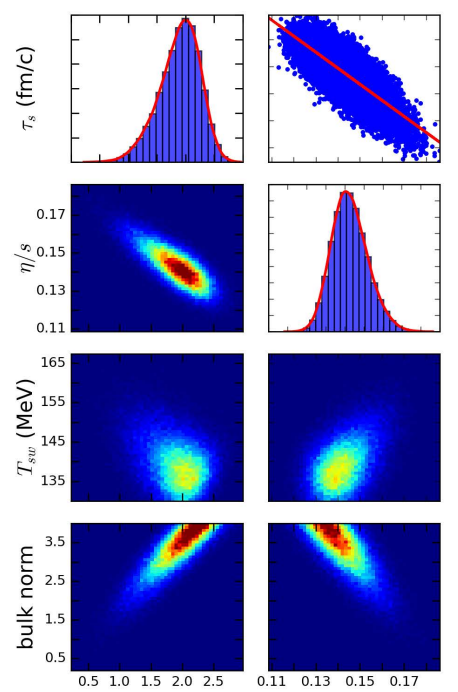

$\tau_{s}(\mathrm{fm} / \mathrm{c})$
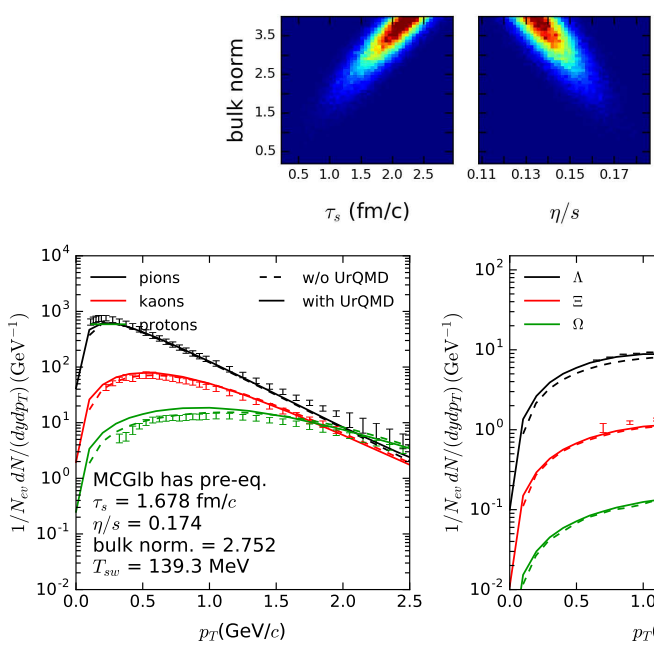

$\eta / s$

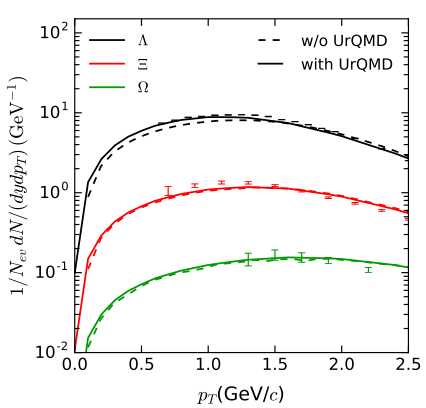

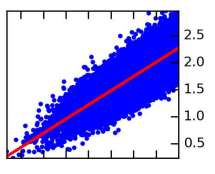
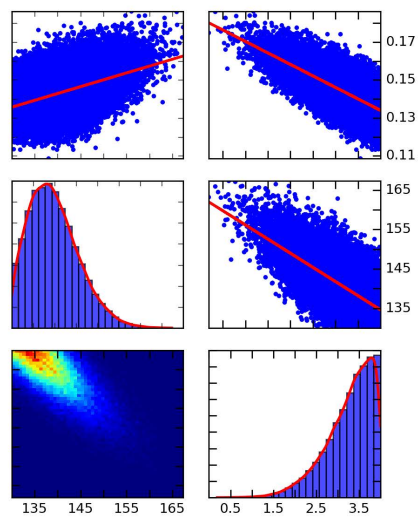

bulk norm

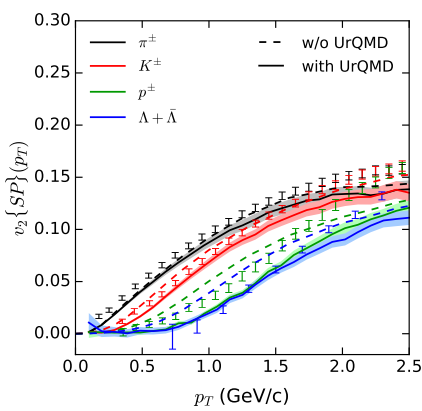

Fig. 1. Upper panel: Likelihood distributions for each of the four fit parameters are shown on the diagonal, correlations between them in upper right triangle of plots, and 2-dimensional projections of the likelihood distributions for pairs of parameters (with dark red indicating the most likely regions) in the lower triangle of plots. Lower panels: $p_{T}$ distributions for pions, kaons and protons (left), $\Lambda, \Xi$ and $\Omega$ (middle), and the differential elliptic flows for $\pi, K, p$ and $\Lambda$ (right) from 400 hydrodynamic simulations with fluctuating initial conditions, each oversampled 400 times with URQMD to obtain sufficient particle statistics. Solid lines: full VISHNU runs. Dashed lines: pure hydro runs with immediate freeze-out at $T_{\mathrm{sw}}$, without subsequent hadronic rescattering using URQMD. Experimental data from ALICE [11].

These tendencies are illustrated in the top panel of Fig. 1 for which we ran VISHNU simulations with smooth ensemble-averaged MC-Glauber initial conditions for $10-20 \%$ central $\mathrm{Pb}-\mathrm{Pb}$ collisions at $2.76 \mathrm{~A} \mathrm{GeV}$ for 1000 different quadruplets of the parameters listed in Table 1, calculated the experimental observables listed in the same table (chosen for their insensitivity to initial-state event-by-event fluctuations), determined the $\chi^{2}$ for each quadruplet by comparing them with the experimental values, and then reconstructed the posterior likelihood distributions in the 4-dimensional parameter space using MCMC sampling [13]. For the set with the lowest $\chi^{2}$, listed in the lower left panel of Fig. 1, we then ran 400 event-by-event VISHNU simulations with fluctuating initial conditions, each oversampled 400 times in the URQMD stage for suffi- 
cient particle statistics, to predict the full transverse momentum distributions and their elliptic flows $v_{2}\left(p_{T}\right)$ for the specific hadron species shown in the bottom row of Fig. 1.

\begin{tabular}{lclllll}
\hline \hline$\left\langle v_{2}^{\text {ch }}\right\rangle[14]$ & $0.0782 \pm 0.0019$ & & & & \\
$\left\langle\left\langle_{3}^{\text {ch }}\right\rangle[14]\right.$ & $0.0316 \pm 0.0008$ & & & & \\
$\left\langle p_{T}\right\rangle_{\pi^{+}}(\mathrm{GeV} / c)[15]$ & $0.542 \pm 0.018$ & & parameter & best & mean & $95 \%$ C.I. \\
$\left\langle p_{T}\right\rangle_{K^{+}}(\mathrm{GeV} / c)[15]$ & $0.825 \pm 0.028$ & & $\tau_{s}(\mathrm{fm} / c)$ & 2.233 & 1.889 & $1.187-2.591$ \\
$\left\langle p_{T}\right\rangle_{p}(\mathrm{GeV} / c)[15]$ & $1.311 \pm 0.043$ & $\eta / s$ & 0.135 & 0.143 & $0.124-0.161$ \\
$\left\langle p_{T}^{2}\right\rangle_{p}\left(\mathrm{GeV}^{2} / c^{2}\right)[15]$ & $2.085 \pm 0.070$ & & $T_{\mathrm{sw}}(\mathrm{MeV})$ & 133.4 & 134.0 & $128.5-151.1$ \\
$\left(d N_{\pi^{+}} / d y\right) /\left(d N_{K^{+}} / d y\right)[15]$ & $6.691 \pm 0.670$ & & bulk norm. & 3.998 & 3.277 & N/A \\
$\left(d N_{\pi^{+}} / d y\right) /\left(d N_{p} / d y\right)[15]$ & $21.667 \pm 2.292$ & & & & & \\
$\left(d N_{\pi^{+}} / d y\right) /\left(d N_{\Lambda} / d y\right)[15]$ & $26.765 \pm 3.639$ & & & & & \\
\hline \hline
\end{tabular}

Table 1. Left: Measured values of nine hadronic observables from 2.76 A TeV $10-20 \%$ central $\mathrm{Pb}+\mathrm{Pb}$ collisions used to constrain the model parameters listed on the right. $\left\langle p_{T}\right\rangle$ and $\left\langle p_{T}^{2}\right\rangle$ are truncated means calculated from the data tables for the transverse momentum distributions listed in [15]. Right: Best-fit parameters and 95\% confidence intervals (C.I.) using the results from Markov Chain Monte Carlo sampling of the posterior parameter distribution shown in Fig. 1 (top).

To illustrate the effect of the microscopically simulated late hadronic rescattering stage on these observables we also added dashed lines illustrating their state at the end of the fluid stage at $T_{\mathrm{sw}}$. Protons and $\Lambda \mathrm{s}$ experience a significant radial boost from URQMD, pushing their yields and elliptic flows to larger transverse momenta. Since $\Lambda_{\mathrm{s}}$ in $\mathrm{UR}_{\mathrm{R}} \mathrm{QMD}$ scatter with reduced cross sections relative to those of protons, this shift towards larger $p_{T}$ is weaker for $\Lambda \mathrm{s}$ than for protons; as a result, the hydrodynamically predicted elliptic flow mass ordering between protons and $\Lambda \mathrm{s}$ at $T_{\mathrm{sw}}$, clearly visible in the dashed lines in the lower right panel of Fig. 1 and also in the experimental data [11], is basically eliminated after hadronic rescattering, although not inverted as previously observed in the VISHNU model without pre-equilibrium dynamics [16]. A shorter lifetime of the hadronic rescattering stage could help to keep this from happening.

\section{References}

[1] C. Shen, Z. Qiu, H. Song, J. Bernhard, S. Bass and U. Heinz, Comput. Phys. Commun. 199 (2016) 61.

[2] U. Heinz et al., Nucl. Phys. A, in press [arXiv:1509.05818 [nucl-th]].

[3] P. F. Kolb and R. Rapp, Phys. Rev. C 67 (2003) 044903; P. F. Kolb, PhD thesis, Universität Regensburg, 2002.

[4] W. Broniowski, W. Florkowski, M. Chojnacki and A. Kisiel, Phys. Rev. C 80 (2009) 034902.

[5] B. Schenke, P. Tribedy and R. Venugopalan, Phys. Rev. Lett. 108 (2012) 252301.

[6] W. van der Schee, P. Romatschke and S. Pratt, Phys. Rev. Lett. 111 (2013) 222302.

[7] P. Romatschke, Eur. Phys. J. C 75 (2015) 429.

[8] J. Liu, C. Shen and U. Heinz, Phys. Rev. C 91 (2015) 064906.

[9] A. Bazavov et al. [HotQCD Collaboration], Phys. Rev. D 90 (2014) 094503.

[10] G. S. Denicol, T. Kodama, T. Koide and P. Mota, Phys. Rev. C 80 (2009) 064901.

[11] R. Preghenella [ALICE Collaboration], arXiv:1203.5904 [hep-ex]; B. B. Abelev et al. [ALICE Collaboration], JHEP 1506 (2015) 190; Phys. Rev. Lett. 111 (2013) 222301; Phys. Lett. B 728 (2014) 216.

[12] K. Dusling and T. Schäfer, Phys. Rev. C 85 (2012) 044909.

[13] C. Quammen, H. Canary, R. M. Taylor II, S. Pratt and J. Wyka https://github.com/MaDAI/ DistributionSampling/blob/master/doc/manual/

[14] G. Aad et al. [ATLAS Collaboration], JHEP 1311 (2013) 183.

[15] B. B. Abelev et al. [ALICE Collaboration], Phys. Rev. C 88 (2013) 044910; Phys. Rev. Lett. 111 (2013) 222301.

[16] X. Zhu, F. Meng, H. Song and Y. X. Liu, Phys. Rev. C 91 (2015) 034904. 